\title{
Temperament and Anxiety: The Mediating Role of Metacognition
}

\author{
Malgorzata Dragan • Wojciech Dragan
}

Published online: 5 October 2013

(C) The Author(s) 2013. This article is published with open access at Springerlink.com

\begin{abstract}
The present study examines a simple model for the relationship between temperament, anxiety and maladaptive metacognition. A clinical sample of patients diagnosed with anxiety disorders $(n=216)$ completed a set of self-reported questionnaires measuring temperament dimensions, state anxiety and metacognitions. Three temperament traits were included in the hypothesized model: emotional reactivity, perseveration and briskness. A structural equation modeling analysis supported a model in which the relationship between the three temperament traits and anxiety were fully mediated by metacognition. Dissimilar models were identified for the male and female subgroups, and also with reference to individual categories of maladaptive metacognition. The findings support the significance of metacognition as a factor influencing the temperament-anxiety relationship. Moreover, they confirm the roles both of emotional reactivity and of perseveration, being major traits related to anxiety which also turned out to be strongly associated with metacognition. In case of the models for the categories of metacognition, emotional reactivity was associated with negative beliefs, perseveration with negative and positive beliefs, while briskness predicted anxiety independently of metacognition. These results suggest the existence of more specific associations between temperament traits, anxiety, and various types of metacognition.
\end{abstract}

Keywords Temperament · Metacognition · Anxiety · Self-regulatory executive function model $\cdot$ Structural equation modeling

Metacognition is a core concept in the metacognitive model of psychopathology and psychotherapy, a relatively new approach

\footnotetext{
M. Dragan $(\bowtie) \cdot$ W. Dragan

Faculty of Psychology, University of Warsaw, ul. Stawki 5/7, 00-183 Warsaw, Poland

e-mail: mpolak@psych.uw.edu.pl
}

which has grown out of the cognitive-behavioural tradition (Wells 2000, 2009). Metacognition is defined as the aspect of cognitive processing being responsible for the monitoring, evaluation, interpretation and regulation of the content of cognitions, and also for its own organization (Moses and Biard 1999). It is assumed that since metacognition fulfils an executive function with regard to cognitive processing (Wells 2000, 2009), it also plays a contributory role in emotional regulation. Wells and Matthews $(1994,1996)$ have proposed the SelfRegulatory Executive Function (S-REF) model, in which dysfunctional metacognition plays a central role in the development and maintenance of major forms of emotional disorder. The authors claim that maladaptive metacognitions play a leading role in emotional regulation and are responsible for the maintenance of a specific style of cognitive processing, attention focusing and coping behaviours, together termed cognitive-attentional syndrome (CAS). It is for this reason that in metacognitive therapy more attention is paid to individuals' patterns of thinking and of directing attention, rather than to the specific content of their thoughts (which is inconstant and often volatile). CAS is seen as constituting a "common core" of various categories of emotional disorder. Through its association with extended thinking about past or future issues (rumination, worrying), the focusing of attention upon threats, and also with the selection of coping strategies such as avoidance or assurance-seeking, it has the effect of prolonging the negative emotional states being the symptoms of emotional disorders. Maladaptive metacognition and other symptoms of CAS have been found to be positively associated with various psychological constructs, such as state or trait anxiety (Irak and Tosun 2008; Spada et al. 2010), also with the symptoms of a range of emotional disorders (e.g. Hermans et al. 2003; Roussis and Wells 2006).

Temperament as one of the basic factors contributing to the development of vulnerability to psychopathology, has a long history of scientific study (Taylor et al. 2009). Temperament can be viewed as a sub-class of personality traits: a relatively 
stable tendency to react to situations in a particular way (Buss and Plomin 1984; Strelau 1983). In most theories of temperament/personality, it has been viewed as a biologically based disposition, influencing behaviour and emotion from early childhood, being observable throughout the individual's life span, and constituting a fundamental factor in psychological adjustment (e.g. Rothbart et al. 2000). A relationship between, on the one hand, sets of similar characteristics included in the main theories of temperament and being classifiable as "anxious temperaments" (i.e. fearfulness, neuroticism, negative affectivity) and, on the other hand, state anxiety and the symptoms of anxiety disorders has already been demonstrated by researchers (e.g. Rettew et al. 2006; Strelau and Zawadzki 2011). Supporting such findings, behaviour genetics studies (e.g. Dragan and Oniszczenko 2009; Oniszczenko and Dragan 2005) have shown that temperament traits being related to anxiety may share certain genetic underpinnings with the symptoms of anxiety disorders. Various psychological theories contain the assumption that anxiety arises on the basis of "anxious traits". The cognitive model, for example, explains this assumption in terms of information processing based on hypervigilance to threat, and also disordered interpretations (Taylor et al. 2009). Meanwhile, Strelau and Zawadzki (2011) have proposed a clarification of the temperament-anxiety relationship with reference to Barlow's model of negative affect dual coping strategies (Barlow 2004). This model emphasises the roles of worry and avoidance: two strategies in effect serving to maintain states of anxiety, and also being consistent with such temperamental tendencies as emotional reactivity or perseveration.

\section{Temperament and Metacognition}

While studies confirm the independent roles of temperament and metacognition in the individual's vulnerability to psychopathology, the relationship between the two constructs has rarely been investigated. Van der Heiden et al. (2010) tested a hierarchical model of vulnerability factors for generalized anxiety disorder (GAD) in a group of 137 patients. The model included general vulnerability factors such as neuroticism and extraversion, and also specific vulnerability factors such as intolerance of uncertainty and metacognitive beliefs (positive and negative). The authors found that negative beliefs mediated the relationship between neuroticism and symptoms of GAD. Dragan et al. (2012) examined a mediation model containing the assumption that metacognitions would mediate the relationship between temperament traits and state anxiety in a student sample; they found a partial mediation effect of metacognition. The authors referred to the Regulative Theory of Temperament (RTT) developed by Strelau (1983, 2008; see also Boyle et al. 2008), distinguishing six temperament traits: briskness, perseveration, sensory sensitivity, emotional reactivity, endurance and activity. RTT states that temperament has the regulative function of moderating the energetic and temporal aspects of all kinds of behaviour, and that individual differences in temperament traits may be observed throughout life, from early infancy onwards (however, most studies on RTT have been conducted upon adults). Rothbart (1989) described the constructs of reactivity and selfregulation from her own theory as being similar to Strelau's conceptualisation, and Strelau and Zawadzki (1995, 2011) showed that RTT dimensions are related to other widely known constructs such as neuroticism and extraversion. A meta-analysis of the research conducted regarding RTT shows that certain of these temperament traits are associated with anxiety, and also suggests that they contribute to psychopathology (Strelau and Zawadzki 2011).

Dragan et al. (2012) in their study ended up focussing on three specific traits, namely emotional reactivity, perseveration and briskness. Emotional reactivity is defined as the tendency to react intensively to emotion-generating stimuli, and is expressed in terms of high emotional sensitivity and low emotional endurance. Perseveration is the tendency to continue and repeat behaviours, and to experience emotional states following a cessation of the stimuli evoking those behaviours or states. Briskness is the tendency to react quickly, maintaining a high tempo in the performing of activities, and easily shifting from one behaviour to another in response to changes in surroundings. Dragan et al. (2012) postulated that those specific temperament traits may play an important role in the development of dysfunctional metacognition. The authors assume that metacognitively directed emotional regulation develops during the process of interaction between dispositional and situational factors. It therefore follows that certain temperament traits may serve as a constitutional basis during this process. An individual becomes prone to psychopathology as a result of the development of maladaptive metacognition, being shaped by unfavourable constitutional characteristics and experiences. Thus, the effects of genetically determined temperament may be transmitted as a result of the nature of volitional mental control processes. For example, emotional reactivity may lead to the development of specific metacognitive strategies aimed at coping with intense emotions. Maladaptive metacognitions learned throughout development, for instance positive beliefs about worry (e.g."worry is helpful in avoiding problems"), may lead to a 'readiness to worry' or 'compulsive' worrying, so extending this type of processing and, in consequence, prolonging a state of anxiety. An undifferentiated trait such as emotional reactivity thus effectively becomes channelled, as a result of being metacognitively directed towards manifesting in certain ways (such as persistent worrying, or the sustained dysfunctional appraisal of internal mental processes), into a vulnerability to pathological anxiety.

To date, the relationship between RTT traits, anxiety and metacognition has been tested in just one study. The primary 
purpose of the present study, given that metacognitive theory refers primarily to psychopathology, was to evaluate the role of metacognition as a potential mediator of the relationship between RTT temperament traits and anxiety, in a clinical sample, and employing the structural equation modeling (SEM) approach. In line with the literature (e.g. Strelau and Zawadzki 2011; Dragan et al. 2012) showing that of all the RTT traits, three in particular - emotional reactivity, briskness and perseveration - were found to be significantly associated with anxiety and symptoms of anxiety disorders, only these were included in the proposed model (emotional reactivity and perseveration were positively related with anxiety, and briskness negatively). Moreover, in order to examine more specific associations, the proposed model was tested separately in groups of males and females, and also with reference to various categories of maladaptive metacognition.

\section{Method}

\section{Participants}

In line with the theoretical assumptions of the S-REF model, stating that maladaptive metacognition is universal among emotional disorders, participants were recruited from among psychiatric patients diagnosed with a range of anxiety disorders. Ultimately, a total sample of 216 participants were recruited, all of whom were undergoing treatment at three Warsawarea State health service psychiatric inpatient clinics treating patients with anxiety and stress-related disorders. One of the admission criteria of these clinics was the ability to participate in psychotherapy. All participants had been diagnosed with an anxiety disorder, as defined in the World Health Organization's International Classification of Diseases (ICD-10), and also had a history of hospitalization due to anxiety disorder. Their diagnoses had been made prior to their current hospitalization, and had been verified by psychiatric interview in the course of their latest pre-hospitalization assessment. The most prevalent diagnosis was mixed anxiety and depressive disorder (38.9\%); $46.7 \%$ of the patients had been diagnosed with anxiety disorder (GAD, panic disorder, agoraphobia or social phobia), while a further $14.4 \%$ of the sample had received a diagnosis of anxiety disorder with a comorbid diagnosis of depressive disorder. The sample contained $55.1 \%$ females and $44.9 \%$ males. The mean age of the female participants was $\mathrm{M}=39.69$ ( $\mathrm{SD}=$ 12.91 , range $=19-71$, while the mean age of the male participants was $\mathrm{M}=43.43$ ( $\mathrm{SD}=8.83$, range $=22-62$ ). The participants were most often married $(48.6 \%$ ), while $26.4 \%$ were single, and $16.0 \%$ were divorced or in formal separation. The proportion in employment amounted to $38.2 \% ; 30.6 \%$ had a disability or old-age pension, and $17.2 \%$ were unemployed. More than half of the sample (55.5\%) had received education to secondary level; $32.0 \%$ had higher education, and $12.5 \%$ only primary education. The participants were informed of the objectives and procedure, and also gave their informed consent prior to the study. The study was approved by the Research Ethics Committee at the Faculty of Psychology, University of Warsaw.

\section{Measures}

In the course of the psychological assessment, a battery of measures was administered to participants, including the following questionnaires:

The Formal Characteristics of Behavior-Temperament Inventory (FCB-TI; Strelau and Zawadzki 1993). This questionnaire includes six dimensions, in line with RTT: briskness, perseveration, sensory sensitivity, emotional reactivity, endurance and activity. Each of the six FCB-TI scales contains 20 items. In line with the theoretical assumptions made, the study focussed on the three dimensions relating to temperament traits: emotional reactivity (ER), briskness (BR) and perseveration (PE). FCB-TI has good psychometric parameters, e.g. Cronbach alphas ranging from .73 to .85 for the subscales representing the six dimensions of RTT (Strelau an Zawadzki 1995). In the current study, Cronbach alphas for the three subscales analysed were as follows: for ER .84, for BR .83, and for PE .71.

The State-Trait Anxiety Inventory (STAI, Spielberger et al. 1970. This is a popular and widely used measure of two forms of anxiety according to Spielberger's theory. State anxiety (X-I) is a measure of current symptoms, whereas trait anxiety (X-II) is a relatively stable personality trait. In line with the theoretical assumptions underlying the current study, the objective was to predict the anxiety state as opposed to trait anxiety, with the aim of assessing current anxiety symptoms and reducing the conceptual and measurement overlap with the more stable, personality and temperamental dimensions being assessed. The Polish adaptation of the scale was performed by Spielberger, Strelau, Tysarczyk and Wrześniewski, and this version of the instrument has very good psychometric properties, e.g. Cronbach's alpha for state anxiety was .84 (Wrześniewski et al. 2002). In the current study, Cronbach's alpha for X-I was .88 .

The Metacognitions Questionnaire-30 (MCQ-30: Wells and Cartwright-Hatton 2004) measures individual differences in the metacognitive beliefs, judgments and monitoring tendencies considered important in the metacognitive model of psychopathology. It consists of five factors: (1) positive beliefs about worry (item example: "Worrying helps me to solve problems"), (2) negative beliefs about thoughts concerning uncontrollability and danger (item example: "When I start worrying I cannot stop"), (3) 
cognitive confidence, i.e. assessing confidence in attention and memory (item example: "I do not trust my memory"), (4) negative beliefs concerning the consequences of not controlling thoughts (item example: "Not being able to control my thoughts is a sign of weakness"), and (5) cognitive self-consciousness, i.e. the tendency to focus attention on thought processes (item example: "I monitor my thoughts"); higher MCQ-30 scores signify a relatively greater the number of dysfunctional metacognitive beliefs being held by the individual. The original version of MCQ30 displays good psychometric properties (Spada et al. 2008; Wells and Cartwright-Hatton 2004). The Polish version of the scale also has good psychometric properties (e.g. Cronbach alphas for the subscales ranged from .70 to $.87 ; \alpha=.87$ for the total scale); furthermore, confirmatory factor analysis has supported the factor solution obtained for the original version (Dragan and Dragan 2011). In the current study, Cronbach's alpha for the general index of MCQ-30 was .87.

\section{Results}

Data analysis was performed using the PASW Statistics 21.0 (SPSS Inc. 2012) and AMOS 21.0 (Arbuckle 2012) software packages. In order to test the relationship between temperament traits, maladaptive metacognition and anxiety, the structural equation modeling technique (SEM) was employed, with the use of the generalized least squares (GLS) method. In SEM, the proposed hypothesised model is evaluated for its goodness of fit with the actual observations in the sample data (Byrne 2010). The following fit indicators were used to evaluate the models: chi-square, chi-square/df ratio, comparative fit index (CFI), root mean square of approximation (RMSEA), generalized fit index (GFI), and Tucker-Lewis index (TLI). The Sobel test was used to determine if the observed mediation effects are significant.

Table 1 shows descriptive statistics for all variables included in the study, for the sample as a whole and also by gender. Comparisons of means by gender revealed statistically significant differences in negative beliefs about thoughts concerning uncontrollability and danger, and also in emotional reactivity (in both cases, females scored significantly higher).

Correlations were calculated between the general index of metacognitions, the individual subscales of metacognitions, state anxiety level, as well as the three temperament traits. The results are summarized in Table 2. Of the three temperament traits two - emotional reactivity and briskness-were correlated both with maladaptive metacognitions and with anxiety (emotional reactivity correlated positively, briskness negatively). In connection with the third trait-perseveration-no such result was observed: it was positively correlated with metacognitions, but not with anxiety.
The proposed structural model of the variables being tested did not fit the data well, either for the whole sample, or for the subgroups of males and females, or in the case of individual categories of metacognition. Figure 1 is a visualization of the identified structural model for the whole sample, being the result of SEM analysis. Standardized estimates are reported. Due to theoretical considerations, correlations between measurement errors for temperament traits were permitted. The fit values for this final model are shown in Table 3. They all fall within acceptable ranges, suggesting a good fit of the model. The three paths - between each of the three temperament traits and anxiety: ER-anxiety, PE-anxiety and BR-anxiety-turned out to be statistically non-significant, and were therefore not included in the final model. These effects of full mediation were significant for both $\operatorname{ER~}(z=3.40, p<.001)$ and $\mathrm{PE}$ $(z=2.04, p<.05)$, but not for $\operatorname{BR}(z=1.78, p=.075)$.

The models identified for the male and female groups with reference to the general index of metacognitions are depicted in Fig. 2. In the model identified for males, two temperament traits were included, namely emotional reactivity and briskness; however, only emotional reactivity was predictive of metacognition $(b=.49)$. Metacognition was significantly predictive for anxiety $(b=.25)$. The ER-anxiety path was not significant and was not included in the final model. This is suggestive of full mediation by metacognition; however, this effect was non-significant $(z=1.86, p=0.06)$. Briskness independently predicted anxiety in this model $(b=-.20)$. Fit indices for the model were as follows-RMSEA: 0.00; CFI: 1.00; $\chi^{2}(2): 1.064(p=.587)$; chi-square degrees of freedom ratio ( $\left.\chi^{2} / \mathrm{df}\right)$ : .532; GFI: 0.994; TLI: 1.066. In the model identified for females, only one temperament trait was included, namely emotional reactivity. It was significantly predictive of metacognition $(b=.50)$, and metacognition was predictive for anxiety $(b=.37)$. The ER-anxiety path was not significant and was not included in the final model; this implies full mediation of metacognition. The mediation effect was significant $(z=$ $3.48, p<.001)$. Fit indices for the female subsample model were as follows-RMSEA: 0.00; CFI: $1.00 ; \chi^{2}(1): .240$ $(p=.625)$; Chi-square degrees of freedom ratio $\left(\chi^{2} / \mathrm{df}\right): .240$; GFI: 0.999; TLI: 1.056 .

Table 3 shows fit indicators for the models identified for the five categories of maladaptive metacognition measured by MCQ-30. All of them suggest a very good fit for the models. In the case of the model for positive beliefs, only perseveration was significantly predictive of this metacognition category $(b=.20)$; positive beliefs were significantly predictive for anxiety $(b=.17)$. The PE-anxiety path was not significant and was not included in the model, implying that full mediation of metacognition was observed (the effect was significant, $z=1.94, p<.05)$. Moreover, in this model, the included independent path, BR-anxiety, was significant $(b=-.20)$.

A similar model was identified for self-consciousness. Perseveration was significantly predictive of this category of 
Table 1 Means and standard deviations for the total sample and by gender $n s$ non-significant

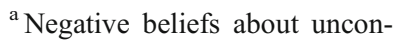
trollability and danger

${ }^{\mathrm{b}}$ Negative beliefs about not controlling thoughts

$* p<0.01$

\begin{tabular}{|c|c|c|c|c|c|c|c|}
\hline \multirow[t]{2}{*}{ Variables } & \multicolumn{2}{|c|}{ Total sample $(n=216)$} & \multicolumn{2}{|c|}{ Women $(n=118)$} & \multicolumn{2}{|c|}{ Men $(n=98)$} & \multirow[t]{2}{*}{$t$} \\
\hline & M & SD & M & $\mathrm{SD}$ & M & SD & \\
\hline State anxiety & 51.20 & 9.78 & 51.53 & 11.50 & 50.66 & 7.19 & $n s$ \\
\hline \multicolumn{8}{|l|}{ Metacognitions } \\
\hline Positive beliefs & 9.87 & 3.98 & 9.69 & 3.83 & 10.06 & 4.19 & $n s$ \\
\hline Negative beliefs- $\mathrm{I}^{\mathrm{a}}$ & 16.66 & 4.49 & 17.39 & 4.12 & 15.75 & 4.81 & $2.68^{*}$ \\
\hline Cognitive confidence & 15.18 & 5.00 & 15.60 & 4.95 & 14.64 & 5.09 & $n s$ \\
\hline Negative beliefs-II ${ }^{\mathrm{b}}$ & 14.17 & 4.35 & 14.36 & 4.38 & 13.84 & 4.27 & $n s$ \\
\hline Self-consciousness & 14.85 & 3.96 & 14.84 & 4.03 & 14.83 & 3.92 & $n s$ \\
\hline General index & 70.73 & 14.92 & 71.87 & 14.01 & 69.13 & 15.97 & $n s$ \\
\hline \multicolumn{8}{|l|}{ Temperamental traits } \\
\hline Emotional reactivity & 14.38 & 4.37 & 15.10 & 3.77 & 13.48 & 4.87 & $2.74 *$ \\
\hline Perseveration & 14.31 & 3.40 & 14.58 & 3.21 & 14.02 & 3.63 & $n s$ \\
\hline Briskness & 11.98 & 4.67 & 11.26 & 4.62 & 12.81 & 4.64 & $n s$ \\
\hline
\end{tabular}

metacognition $(b=.24)$; self-consciousness was significantly predictive for anxiety $(b=.13)$; on the other hand, the PEanxiety path, being non-significant, was not included, suggesting full mediation of metacognition (this effect was however non-significant, $z=1.73, p=.082)$. The included independent path, BR-anxiety, was also significant $(b=-.21)$.

For cognitive confidence, the model identified appears quite different. Two of the three RTT traits were included: emotional reactivity and briskness. Emotional reactivity was predictive of this category of metacognition $(b=.25)$, and metacognitions were significantly predictive of anxiety $(b=.13)$. Again, an effect of full mediation of metacognition was observed (however, this effect was non-significant: $z=1.61, p=.108)$. Briskness, the second trait included in the model, was predictive of cognitive confidence $(b=-.29)$, and the BR-anxiety path was significant $(b=-.17)$, suggesting partial mediation of metacognition (however, this effect was non-significant, $z=1.64, p=.099$ ).

In the case of the next model identified-for negative beliefs regarding uncontrollability and danger- two traits were included: emotional reactivity and perseveration. Both emotional reactivity $(b=.46)$ and perseveration $(b=.14)$ were

Table 2 Correlations between temperament traits, metacognitions and anxiety $(n=216)$

\begin{tabular}{|c|c|c|c|c|c|c|c|c|c|c|}
\hline & \multicolumn{3}{|c|}{ Temperament traits } & \multicolumn{6}{|c|}{ Metacognitions } & \multirow[t]{2}{*}{ Anxiety } \\
\hline & $\mathrm{BR}$ & ER & $\mathrm{PE}$ & GI & PB & NB-I & $\mathrm{CC}$ & NB-II & $\mathrm{SC}$ & \\
\hline \multicolumn{11}{|c|}{ Temperament traits (FCB-TI) } \\
\hline $\mathrm{BR}$ & - & & & & & & & & & \\
\hline ER & $-.48 * *$ & - & & & & & & & & \\
\hline $\mathrm{PE}$ & $-.21 * *$ & $.55^{* *}$ & - & & & & & & & \\
\hline \multicolumn{11}{|c|}{ Metacognitions (MCQ-30) } \\
\hline GI & $-.32 * *$ & $.49 * *$ & $.38 * *$ & - & & & & & & \\
\hline PB & ns & $.16^{*}$ & $.20 * *$ & $.57 * *$ & - & & & & & \\
\hline NB-I & $-.33 * *$ & $.54 * *$ & $.39 * *$ & $.78^{* *}$ & $.25^{* *}$ & - & & & & \\
\hline $\mathrm{CC}$ & $-.42 * *$ & $.39 * *$ & $.15^{*}$ & $.62 * *$ & ns & $.44 * *$ & - & & & \\
\hline NB-II & $-.16^{* *}$ & $.35^{* *}$ & $.32 * *$ & $.80 * *$ & $.40 * *$ & $.51 * *$ & $.32 * *$ & - & & \\
\hline $\mathrm{SC}$ & ns & $.20 * *$ & $.24 * *$ & $.69 * *$ & $.29 * *$ & $.43 * *$ & $\mathrm{~ns}$ & $.53 * *$ & - & \\
\hline \multicolumn{11}{|c|}{ Anxiety (STAI X-I) } \\
\hline & $-.22 * *$ & $.21 * *$ & ns & $.34 * *$ & $.20 * *$ & $.32 * *$ & $.20 * *$ & $.31 * *$ & $.12 *$ & - \\
\hline
\end{tabular}

$B R$ briskness, $E R$ emotional reactivity, $P E$ perseveration, $G I$ general index of metacognitions, $P B$ positive beliefs about worry, $N B-I$ negative beliefs about uncontrollability and danger, $C C$ cognitive confidence, $N B$-II negative beliefs about not controlling thoughts, $S C$ cognitive self-consciousness; FCB-TI The Formal Characteristics of Behavior - Temperament Inventory, MCQ-30 The Metacognitions Questionnaire-30, STAI X-I The State-Trait Anxiety Inventory, $n s$ non-significant

$* p<0.01, * * p<.001$ 


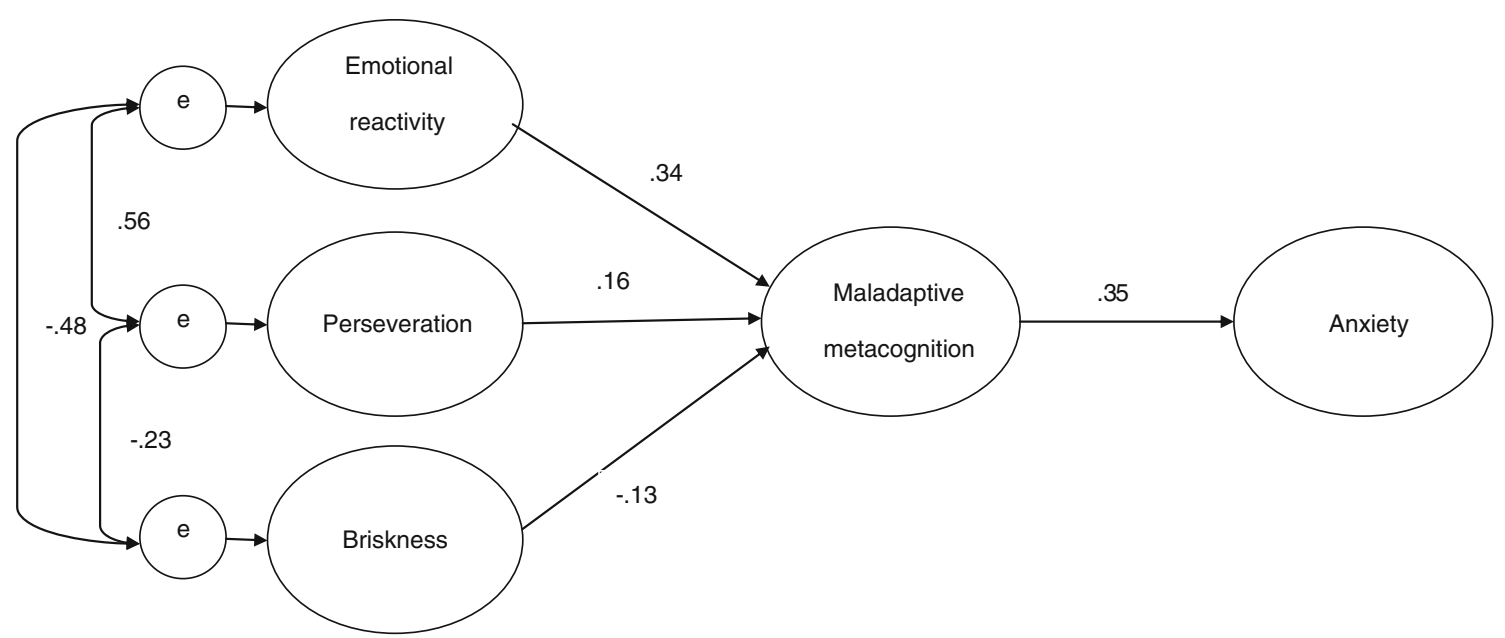

Fig. 1 Structural equation model with the standardized path coefficients between three temperament traits, maladaptive metacognition, and anxiety. Note: $e$ error

predictive of this type of negative belief, which in turn was predictive of anxiety $(b=.32)$. The ER-anxiety and PEanxiety paths were not included, implying full mediation of metacognition, the effects being significant (ER: $z=3.98$, $p<.001$; PE: $z=2.41, p=.016$ ).

In the case of the final model identified-for negative beliefs about not controlling thoughts - in common with the previous category of negative beliefs, both emotional reactivity $(b=.25)$ and perseveration $(b=.18)$ were predictive of this category, which in turn was predictive of anxiety $(b=.28)$. The ER-anxiety and PE-anxiety paths were not included, implying full mediation of metacognition, the effects being significant (ER: $z=2.62, p<.01$; PE: $z=2.09, p<.05$ ). The independent path, BR-anxiety, also identified in this model, was significant $(b=-.18)$.

\section{Discussion}

In the current study we tested a relatively simple model in which, using maladaptive metacognition as an intervening variable, we attempted to clarify the relationships between state anxiety and three RTT traits. The results show metacognition being a significant mediator of the relationship between the three RTT traits and state anxiety, for the entire sample. Despite the fact that at the zero-order level not all the correlations between the analysed variables were significant (perseveration did not correlate with anxiety-Table 2), SEM analysis allowed us to control measurement error correlations for the RTT traits, which was important for theoretical reasons (previous research had shown that a majority of the RTT dimensions intercorrelate; Strelau and Zawadzki 1995); these correlations were relatively high, and influenced the final appearance of the model.

The results of the SEM analysis indicate that a significant mediation effect for metacognition was observed in two out of the three temperament traits: emotional reactivity and perseveration. Namely, effects of full mediation were observed in the identified model (Fig. 1) in both cases (for briskness, the effect of full mediation was not significant at the .05 level, although a tendency was observed). In particular, emotional reactivity-a construct related to other 'anxious traits' or fearfulness (Strelau

Table 3 Summary of fit indicators for models identified for various categories of metacognitions

\begin{tabular}{|c|c|c|c|c|c|c|c|c|}
\hline Model & $\chi^{2}$ & $\mathrm{df}$ & $\mathrm{p}$ & $\chi^{2} / \mathrm{df}$ & CFI & RMSEA & GFI & TLI \\
\hline Metacognitions - general index & 5.000 & 3 & .172 & 1.667 & .976 & .056 & .991 & .921 \\
\hline Positive beliefs & 1.356 & 2 & .508 & .678 & 1.00 & .000 & .997 & 1.088 \\
\hline Negative beliefs- $\mathrm{I}^{\mathrm{a}}$ & 2.367 & 2 & .306 & 1.183 & .995 & .029 & .994 & .984 \\
\hline Cognitive confidence & 1.715 & 1 & .190 & 1.715 & .985 & .058 & .996 & .908 \\
\hline Negative beliefs-II ${ }^{\mathrm{b}}$ & 2.089 & 3 & .554 & .696 & 1.00 & .000 & .996 & 1.035 \\
\hline Self-consciousness & .090 & 2 & .956 & .045 & 1.00 & .000 & 1.00 & 1.218 \\
\hline
\end{tabular}

$d f$ degrees of freedom, CFI comparative fit index, RMSEA root mean square of approximation, GFI generalized fit index, TLI Tucker-Lewis index

${ }^{\text {a }}$ Negative beliefs about uncontrollability and danger

${ }^{\mathrm{b}}$ Negative beliefs about not controlling thoughts 
a)

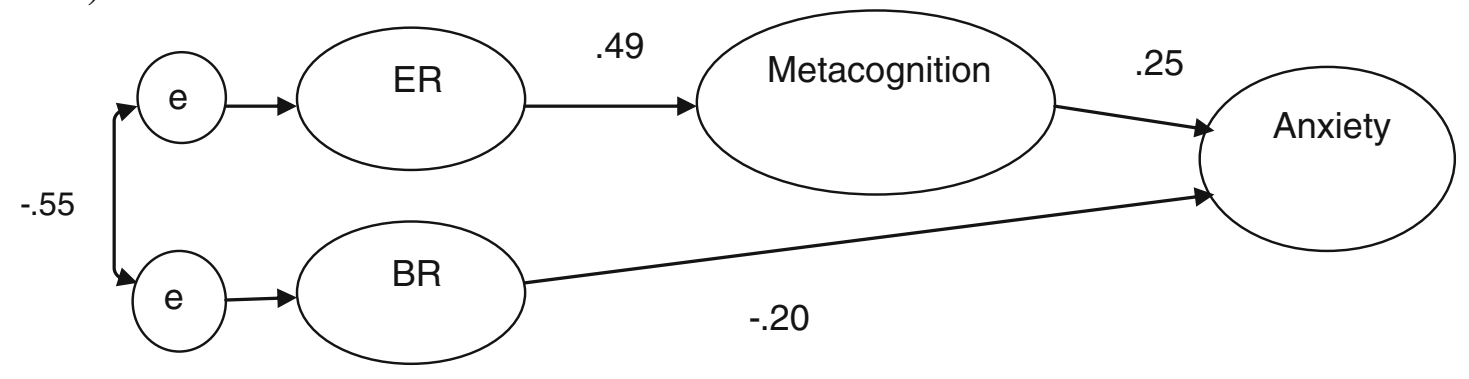

b)

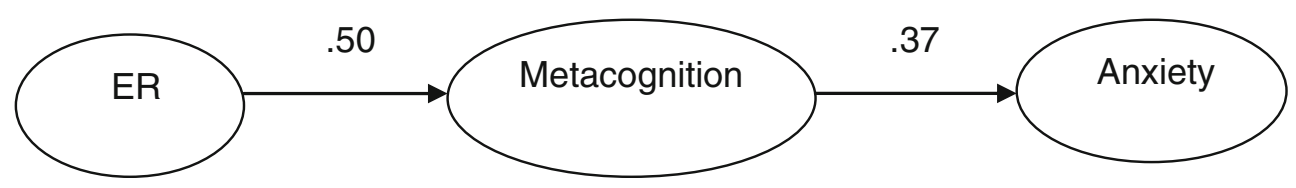

Fig. 2 Structural equation models with the standardized path coefficients identified for the male and female groups. Note. ER emotional reactivity, BR briskness; a model identified for male group, $\mathbf{b}$ model identified for female group. Note: $e$ error

and Zawadzki 2011) — appears to have a primary role in explaining the association between temperament, metacognition and anxiety. This fact was further confirmed in the models identified separately for the male and female subgroups (Fig. 2); (however the effect of full mediation was significant only in the case of the female subgroup). These results can be interpreted with reference to earlier research by van der Heiden et al. (2010), who tested a clinical sample and found that metacognition fully mediated the relationship between neuroticism and the symptoms of anxiety. The present study can therefore be considered a replication of that study, on account of the fact that the RTT traits have been found to correlate also with the 'Big Five' personality factors (Strelau and Zawadzki 1995). Moreover, a comparison of the results from the current study and van der Heiden et al. (2010) with those obtained by Dragan et al. (2012) using a student sample, where effects of partial mediation of metacognition were found, suggests an intensification of maladaptive metacognitions in clinical samples. In the light of metacognitive theory, this can be interpreted as resulting from the specific cognitive processing style observed in patients with emotional disorders. It may be assumed that the psychological functioning of such patients is in some way dominated by cognitive-attentional syndrome symptoms causing, intensifying and prolonging the state of anxiety, and at the same time diminishing the self-reported effects of temperament on their current emotional state.

The present study is the first to have examined the roles played by the various categories of maladaptive metacognition in mediating the relationship between specific temperament traits and anxiety. At a theoretical level, assessing the role of each category of metacognition may help shed light on specific relationships between certain traits and psychopathology. For example, in the case of positive beliefs about worry and cognitive self-consciousness, only one RTT trait- perseveration - was included in the models. Both types of metacognition were found to fully mediate the relationship between perseveration and anxiety; however, this effect was significant only for positive beliefs (in the case of selfconsciousness, a tendency was observed). The effect of mediation may simply result from content agreement between constructs; alternatively, in the light of metacognitive theory, this specific temperament trait may constitute the basis for the development of precisely those types of metacognition. One of the traits (positive beliefs) is linked with the initiation of the worrying cycle (type I worry; Wells 2009), while another (self-consciousness) is linked with an exacerbated tendency to focus the attention on thought processes. Thus, a specific temperament tendency to continue behaviours and experience emotional states despite a cessation of stimuli may reinforce the development of maladaptive metacognition associated with focusing the attention on a specific thought process, so prolonging the experiencing of negative emotions (see also Manfredi et al. 2011).

In the case of all the remaining types of metacognition (cognitive confidence and two types of negative beliefs), emotional reactivity was included in the identified models. In the case of negative beliefs, this is consistent with the earlier findings of van der Heiden et al. (2010), who showed that metacognitive negative beliefs mediated the relationship between neuroticism and symptoms of GAD. However, in the model from the current study, perseveration was additionally included and an effect of full mediation was also obtained. Similarly, these results may be interpreted as an effect of content agreement between the constructs emotional reactivity, perseveration and the two types of negative belief; alternatively, in the light of metacognitive theory, we could speculate that those specific temperament traits constitute the basis for the development of maladaptive metacognitions linked with the 
intensification of anxiety (type II worry; Wells 2009). In the case of cognitive confidence, briskness replaced perseveration in the identified model; however, the effects of mediation turned out to be non-significant. Interestingly, this trait appeared also in the three models identified for specific metacognitions (positive beliefs, cognitive self-consciousness, negative beliefs about uncontrollability and danger), and additionally in the model identified for males, as an independent predictor of anxiety not related to metacognition. Given previous findings that this trait may act as protective factor against emotional disorders (Strelau and Zawadzki 2011; Dragan et al. 2012), further studies are called for to explore the association between this specific trait and anxiety, with reference to other possibly intervening variables not included in our study. For example, Hong (2013) has shown that several specific socialcognitive vulnerability factors such as ruminative style or anxiety sensitivity fully mediate between various dispositional traits and psychopathological symptoms. Since metacognition is a higher-order construct relative to specific cognitive processing mechanisms, it would be worth adopting a broader view, including both cognitive and metacognitive vulnerability factors, in future research.

The results of our study also raise the issue of the development process for maladaptive metacognition. The principal weakness of this study lies in the cross-sectional nature of its design, which effectively restricts the drawing of conclusions regarding causality. There is a need to conduct longitudinal studies enabling an examination of the causal role of various variables and patterns of reciprocal causality. These should include not only dispositional factors such as temperament traits, but also raise the issue of the biological determinants and environmental factors possibly influencing the process of metacognition development. The models and variables presented here, though theoretically based, are correlational in nature. The data could also be used to support the explanation that the measures of temperament traits and metacognition simply reflect the presence of an emotional disorder, or else that temperament traits and metacognition are simply variables being correlated with anxiety. A further important problem is that this study focused on metacognitive beliefs only, and not on other symptoms comprising the cognitiveattentional syndrome. Future studies should include measures of different aspects of this syndrome (e.g. attentional biases) in order to determine its distinctness from temperamental factors. Also, for the same reasons future studies should examine behavioural and neurological demonstrations of temperament. It would also be worthwhile, in the search for specific associations between vulnerability factors and symptomatology, to include various categories of anxiety disorders in hypothesized models.

The results of the present study can however be treated as providing evidence for the metacognitive theory of psychopathology: they strengthen the validity of metacognition not only as an important trait variable, but also as a significant mediator in the relationship between temperament and pathological anxiety. This supports the general aims of metacognitive psychotherapy, directed towards the attenuation and modification of maladaptive metacognition. Moreover, the current study has shed some light on individual relationships between specific temperament traits and categories of metacognition, as predictors of anxiety. It may be hypothesized that specific types of metacognition arise on the basis of specific temperament traits; however, future research, employing a developmental perspective, is needed in order to test this hypothesis. The current results also suggest that future studies should cover the issue of the various determinants of metacognition, so affording a better understanding of the differences between healthy individuals and those diagnosed with emotional disorders.

Acknowledgments Study was supported by internal funds of the Faculty of Psychology at University of Warsaw, donation number: BST154543.

Open Access This article is distributed under the terms of the Creative Commons Attribution License which permits any use, distribution, and reproduction in any medium, provided the original author(s) and the source are credited.

\section{References}

Arbuckle, J. L. (2012). AMOS 21.0. Crawforville: Amos Development Corporation.

Barlow, D. H. (2004). Anxiety and its disorders. The nature and treatment of anxiety and panic (2nd ed.). New York: The Guilford Press.

Boyle, G. J., Matthews, G., \& Saklofske, D. H. (2008). The SAGE handbook of personality theory and assessment. London: SAGE Publications.

Buss, A. H., \& Plomin, R. (1984). Temperament: Early developing personality traits. Hillsdale: Erlbaum.

Byrne, B. M. (2010). Structural equation modelling with AMOS. Hove: Routledge.

Dragan, M., \& Dragan, W. (2011). Właściwości psychometryczne polskiej wersji The Metacognitions Questionnaire-30 [Psychometric properties of the Polish version of the Metacognitions Questionnaire-30]. Psychiatria Polska, 4. Retrived from http://www.psychiatriapolska.pl.

Dragan, W. Ł., \& Oniszczenko, W. (2009). The association between dopamine D4 receptor exon III polymorphism and intensity of PTSD symptoms among flood survivors. Anxiety, Stress and Coping, 22, 483-495.

Dragan, M., Dragan, W., Kononowicz, T., \& Wells, A. (2012). On the relationship between temperament, metacognition and anxiety: independent and mediated effects. Anxiety, Stress and Coping, 6, 697-709.

Hermans, D., Martens, K., De Cort, K., Pieters, G., \& Eelen, P. (2003). Reality monitoring and metacognitive beliefs related to cognitive confidence in obsessive-compulsive disorder. Behaviour Research and Therapy, 41, 383-401.

Hong, R. Y. (2013). From dispositional traits to psychopathological symptoms: social-cognitive vulnerabilities as intervening mechanisms. Journal of Psychopathology and Behavioral Assessment, in press.

Irak, M., \& Tosun, A. (2008). Exploring the role of metacognition in obsessive-compulsive and anxiety symptoms. Journal of Anxiety Disorders, 8, 1316-1325. 
Manfredi, C., Caselli, G., Rebecchi, D., Rovetto, F., Ruggiero, G. M., Sassaroli, S., \& Spada, M. M. (2011). Temperament and parental styles as predictors of ruminative brooding and worry. Personality and Individual Differences, 50, 186-191.

Moses, L. J., \& Biard, J. A. (1999). Metacognition. In R. A. Wilson \& F. C. Keil (Eds.), The MIT encyclopedia of the cognitive sciences (pp. 533-535). Cambridge: MIT Press.

Oniszczenko, W., \& Dragan, W. Ł. (2005). Association between the dopamine D4 receptor gene polymorphism and emotional reactivity as a temperamental trait. Twin Research and Human Genetics, 8, 633-637.

Rettew, D. C., Doyle, A. C., Kwan, M., Stanger, C., \& Hudziak, J. J. (2006). Exploring the boundary between temperament and generalized anxiety disorder: a receiver operating characteristic analysis. Journal of Anxiety Disorders, 7, 931-945.

Rothbart, M. K. (1989). Temperament in childhood: a framework. In G. Kohnstamm, J. Bates, \& M. K. Rothbart (Eds.), Temperament in childhood (pp. 59-73). Chichester: Wiley.

Rothbart, M. K., Ahadi, S. A., \& Evans, D. E. (2000). Temperament and personality: origins and outcomes. Journal of Personality and Social Psychology, 78, 122-135.

Roussis, P., \& Wells, A. (2006). Posttraumatic stress symptoms: tests of relationships with thought control strategies and beliefs as predicted by the metacognitive model. Personality and Individual Differences, 40, 111-122.

Spada, M. M., Mohiyeddini, C., \& Wells, A. (2008). Measuring metacognitions associated with emotional distress: factor structure and predictive validity of the metacognitions questionnaire 30 . Personality and Individual Differences, 45, 238-242.

Spada, M. M., Georgiou, G. A., \& Wells, A. (2010). The relationship among metacognitions, attentional control, and state anxiety. Cognitive Behaviour Therapy, 39, 64-71.

Spielberger, C. D., Gorsuch, R. L., \& Lushene, R. E. (1970). Manual for the state-trait anxiety inventory. Palo Alto: Consulting Psychologists Press.

SPSS Inc. (2012). PASW statistics 21.0. Chicago: SPSS Inc.

Strelau, J. (1983). Temperament, personality, activity. London: Academic.
Strelau, J. (2008). Temperament as a regulator of behavior: After 50 years of research. Clinton Corners: Eliot Werner Publications.

Strelau, J., \& Zawadzki, B. (1993). The Formal Characteristics of Behaviour - Temperament Inventory (FCB-TI): theoretical assumptions and scale construction. European Journal of Personality, 7 , 313-336.

Strelau, J., \& Zawadzki, B. (1995). The Formal Characteristics of Behaviour - Temperament Inventory (FCB-TI): validity studies. European Journal of Personality, 9, 207-229.

Strelau, J., \& Zawadzki, B. (2011). Fearfulness and anxiety in research on temperament: temperamental traits are related to anxiety disorders. Personality and Individual Differences, 7, 907-915.

Taylor, S., Abramowitz, J. S., McKay, D., \& Asmundson, G. J. G. (2009). Anxious traits and temperaments. In D. J. Stein, E. Hollander, \& B. O. Rothbaum (Eds.), Textbook of anxiety disorders (2nd ed., pp. 7386). Washington, D.C.: American Psychiatric Publishing, Inc.

Van der Heiden, C., Melchior, K., Muris, P., Bouwmeester, S., Bos, A. E. R., \& van der Molen, H. T. (2010). A hierarchical model for the relationships between general and specific vulnerability factors and symptom levels of generalized anxiety disorder. Journal of Anxiety Disorders, 24, 284-289.

Wells, A. (2000). Emotional disorders and metacognition: Innovative cognitive therapy. Chichester: Wiley.

Wells, A. (2009). Metacognitive therapy for anxiety and depression. New York: Guilford Press.

Wells, A., \& Cartwright-Hatton, S. (2004). A short form of the metacognitions questionnaire: properties of the MCQ-30. Behaviour Research and Therapy, 42, 385-396.

Wells, A., \& Matthews, G. (1994). Attention and emotion: A clinical perspective. Hillside: Erlbaum.

Wells, A., \& Matthews, G. (1996). Modeling cognition in emotional disorder: the S-REF model. Behaviour Research and Therapy, 34, 867-870.

Wrześniewski, K., Sosnowski, T., \& Matusik, D. (2002). Inwentarz Stanu i Cechy Lęku - Polska adaptacja STAI - Podręcznik [STAI Manual]. Warszawa: PTP. 